\title{
An empirical approach to demographic inference with genomic data
}

\author{
Peter L. Ralph
}

April 2, 2019

\begin{abstract}
Inference with population genetic data usually treats the population pedigree as a nuisance parameter, the unobserved product of a past history of random mating. However, the history of genetic relationships in a given population is a fixed, unobserved object, and so an alternative approach is to treat this network of relationships as a complex object we wish to learn about, by observing how genomes have been noisily passed down through it. This paper explores this point of view, showing how to translate questions about population genetic data into calculations with a Poisson process of mutations on all ancestral genomes. This method is applied to give a robust interpretation to the $f_{4}$ statistic used to identify admixture, and to design a new statistic that measures covariances in mean times to most recent common ancestor between two pairs of sequences. The method more generally interprets population genetic statistics in terms of sums of specific functions over ancestral genomes, thereby providing concrete, broadly interpretable interpretations for these statistics. This provides a method for describing demographic history without simplified demographic models. More generally, it brings into focus the population pedigree, which is averaged over in model-based demographic inference.
\end{abstract}

\section{Introduction}

For most of the history of the field, geneticists made great progress with data that comprise a tiny fraction of all that is in the genome. Even the hundreds of thousands of markers on modern genotyping chips make certain inferences impossible due to ascertainment of these markers. Today, whole-genome sequence is being used to make striking new discoveries about health and human history [e.g. Bycroft et al., 2017, Haak et al., 2015]; such data will soon be commonplace as well in nonmodel organisms. The scale and unbiased nature of these data opens up new opportunities, but will also require new methods, as many assumptions made previously may no longer be necessary.

To do this, it is helpful to begin with the process that generated these data. The rules by which the majority of genetic material is inherited by diploid, sexually reproducing organisms are simple: each new individual carries two distinct copies of the genome - one from mom, one from dad - and each of these copies is formed by recombining different parts of the parent's two copies so as to make a whole. The inherited copies may be modified by mutation, which provides the raw material not only for evolution, but also the means for us to learn about those genomes' histories.

The network describing parent-offspring relationships between past and present members of a population is known as the population pedigree; adding an encoding of the genetic relationships - which parts of each genome was inherited from which copy of the parental genomes - produces the ancestral recombination graph [ARG, described by Griffiths and Marjoram, 1997]. Supposing that any particular position in one of those copies of the genome was inherited from a single one of the two ancestral copies - for the most part true then at each point on the genome these genetic relationships form a tree (known as the gene tree or gene genealogy) that is embedded within the larger pedigree. This information can be efficiently represented as a tree sequence [Kelleher et al., 2016], and can now be produced on a genomic scale from population simulations [Kelleher et al., 2018, Haller et al., 2018]. Just as the population pedigree can be thought of as constraining the collection of gene trees, so the ancestral recombination graph constrains the patterns of concordance and discordance of genetic variants created by mutation within each population. 
Inference with population genetic data usually works by specifying a population model - e.g., a randomly mating population of fixed size $N$ - that determines a probability distribution on the population pedigree, and then assuming that mutation and recombination occurs independently within this [Ewens, 2004]. This paper deals with the intermediate layer - if we take the population pedigree, or even the ancestral recombination graph, as fixed, then what can we learn about it? What are the statistics of population genetics telling us about it? This is partly a matter of philosophy: should the population pedigree that has actually occurred be treated as an instantiation of a random process, with the goal of inferring parameters of that process? Or, is it better to treat the population pedigree as a nonrandom but complex and partially observed object that we wish to estimate summary statistics of? The former view may be most appropriate if we have one or several well-defined demographic models we believe are close to the truth, but the latter is useful for making robust inferences in the absence of any realistic demographic model, as well as for identifying whether we have power to distinguish such models.

The approach of this paper is simple: Assuming that mutations occur independently of demography and recombination (and are hence neutral), these can be treated as a Poisson process on the set of all ancestral genomes, and most population genetics statistics can be written as integrals of specific functions against this Poisson process. By treating the ancestral recombination graph as fixed, the means and variances of these statistics can be found in terms of integrals of the same functions against the mutation rate, and can therefore be interpreted as descriptive statistics of the unknown, but real, ancestral recombination graph. Stochastic models of population genetics generally include demography, recombination, and mutation: this paper takes the outcomes of the first two as fixed, dealing only with the randomness of mutation. More information is obtainable by also treating recombination as random, but it is useful to begin with only mutation, especially as recombination itself is not directly observed, and many commonly used population genetics statistics do not model recombination (e.g., the site frequency spectrum).

The remainder of this paper is structured as follows. After a review of some of the literature, two main results are presented in non-technical terms, given for their inherent interest as well as examples of the general method of calculation that is presented in the subsequent section. Next come proofs of the main results, followed by some more discussion of the results and future work.

\section{Background}

The main point of this paper is to see what can be learned about the population pedigree, or more properly the ARG, by taking an empirical point of view, i.e., treating it as a fixed, but unknown object. Others have taken this approach, albeit usually less explicitly. Slatkin [1991] interprets probabilities of identity by descent and $F_{S T}$ as summaries of the underlying gene trees; the discussion is framed in terms of randomly mating populations, but much of the discussion is applicable without thinking of the trees as random. McVean [2002, 2009] continued in this vein, explaining what two common population genetics statistics, linkage disequilibrium and the principal components decomposition, have to say about these trees. Wakeley et al. [2012] begins from a similar philosophical point, that the pedigree should be treated as a fixed parameter, and goes on to show that data generated under a fixed pedigree differ very little from those generated under a standard coalescent model (which does not incorporate correlations due to a fixed pedigree).

Formal methods describing expectations of gene transmission in a known pedigree have long been used by plant and animal breeders. In particular, Wright [1922]'s method of "path coefficients" treated the pedigree as a known object, averaging over recombination and segregation. These methods are related to a parallel issue arising in genetic association studies [reviewed by Speed and Balding, 2014] where relatedness of samples is a major confounding factor, and alternative approaches can be seen as estimating the actual degree of genetic relatedness or as averaging over it.

The empirical point of view is much more natural in the context of phylogenetics, where between most groups of species, most loci have nearly the same genealogical relationship, and the goal is to directly infer this common tree. For instance, Gillespie and Langley [1979] pointed out that variation in coalescence time contributed to variance in between-species divergence, and Edwards and Beerli [2000] further partition the variance in divergence into components induced by the substitution process and by the demographic process. Similarly, studies of nonrecombining loci often focus on the single underlying tree. The tree that 
describes relationships between, say, mitochondria provides a valuable look into population history and dynamics [Avise et al., 1987, Templeton et al., 1995], and is sometimes of independent interest [Cann et al., 1987, Vigilant et al., 1991]. Tang et al. [2002] studied the "frequentist" approach to estimating time to most recent common ancestor (TMRCA) for modern human Y-chromosomes. Hudson [2007] continued this work, finding the variance of Thomson et al. [2000]'s TMRCA estimator (which is based on the density of segregating derived mutations). Here, we trade precise knowledge of one tree for vague knowledge about many, and are aided by the theoretical tools of phylogenetics [Semple and Steel, 2003].

Early examples of using whole-genome data to estimate an empirical quantity of the population pedigree were the inferences of gene flow between diverged species by Kulathinal et al. [2009] (between Drosophila species), and by Green et al. [2010] (Neanderthal to modern humans). A wider family of similar statistics were subsequently developed in the human population genetics literature [e.g., Durand et al., 2011, Patterson et al., 2012], and have formed the basis for many new insights into human history and evolution. In recent years, a substantial body of work has used these statistics, often applied to ancient DNA from ancient hominid remains, to add new dimensions to our understanding of how modern humans evolved and colonized the globe [e.g., Damgaard et al., 2018, McColl et al., 2018]. One of the main tools in this work is the $f_{4}$ statistic, which is studied in detail below. Many properties of these statistics are described in Peter [2016], but the general behavior of these statistics under complex models of population history remains unclear. Understanding these in a more general context was a major impetus for the present work.

\section{Main results}

The purpose of this paper is to outline a general method of calculation, the utility of which is best communicated with examples. First, a few assumptions. I assume that at each site there are no more than two variants seen (the proportion of triallelic sites within populations is extremely small, but see Jenkins and Song [2011]); and that at each site a reference allele has been chosen; "allele frequencies" are frequencies of the other, alternate allele. However, all statistics developed here are invariant under relabeling of alleles.

I also make the standing assumption of low per base pair mutation rate - precisely, the infinite sites model of mutation [Watterson, 1975], under which each new mutation occurs at a distinct site, and is hence observable. This assumption is quite common in population genetics, where the typical time separating samples is small enough to make this assumption a good one (but I return to this below).

As a first example, consider nucleotide diversity, which for a set $X$ of sampled genomes is denoted $\pi(X)$ and is defined to be the mean proportion of genotyped sites at which two genomes chosen randomly, without replacement from $X$ differ. If each mutation occurs at a unique site, two genomes $x$ and $y$ differ at a site if and only if there has been a mutation at that site in some ancestor from which either $x$ or $y$, but not both, has inherited that site. In other words, sites that contribute to $\pi(X)$ through the comparison of $x$ and $y$ are those at which a mutation has occurred on the branches leading from $x$ and $y$ back to their common ancestor. If mutations occur with probability $\mu$ per generation and per site, nucleotide diversity is therefore an estimator of the mean time to most recent common ancestor - more precisely, $\pi(X) \approx 2 \mu \bar{t}_{X}$, where $\bar{t}_{X}$ is the number of generations from two random samples from $X$ back to their common ancestor, averaged over choices of samples and sites in the genome. How precisely does $\pi(X)$ estimate this mean time to most recent common ancestor? Suppose for simplicity that there are only two genomes in the set $X$. Randomness only enters through the addition of mutations: if we compute $\pi(X)$ averaging across $G$ sites, then $G \pi(X)$ is Poisson with mean $2 \mu \bar{t}_{X} G$; and so the standard deviation of $\pi(X)$ is $\sqrt{2 \mu \bar{t}_{X} / G}$. Genomic data should give very accurate estimates of $\bar{t}_{X}$ in regions where the mutation rate is constant.

Next, let's apply the same intuition to the $f_{4}$ statistic [Patterson et al., 2012, Peter, 2016]: if $X, Y, U$, and $V$ are four sets of sampled genomes, and $p_{X}(\ell)$ is the allele frequency among the samples in $X$ at site $\ell$, etcetera, then the $f_{4}$ statistic of these samples is

$$
f_{4}(X, Y ; U, V)=\frac{1}{G} \sum_{\ell=1}^{G}\left(p_{X}(\ell)-p_{Y}(\ell)\right)\left(p_{U}(\ell)-p_{V}(\ell)\right)
$$


where the sum is over some set of $G$ sites. Notice that $f_{4}(X, Y ; U, V)=-f_{4}(Y, X ; U, V)$. This implies that if the relationship of $X$ and $Y$ to $U$ and $V$ is symmetric, then the expected value of the statistic is zero. In particular, it is zero under a tree-based model of populations, in which $X$ and $Y$ diverged from a common ancestral population more recently than their common ancestors with $U$ or $V$, and there was no subsequent gene flow from $U$ or $V$. Green et al. [2010] and Reich et al. [2010] used these statistics to provide evidence for gene flow from archaic hominids into modern humans. For example, taking $X$ to be a sample of subSaharan Africans, $Y$ a sample of western Europeans, $U$ a Neanderthal, and $V$ a chimpanzee, a significantly negative value implies that European allele frequencies are more correlated with Neanderthal than expected under a model of no admixture; and given archaeological evidence, this supports interbreeding between Neanderthals and modern humans after leaving Africa. However, it is not at first clear how the statistic should perform under more realistic population histories (e.g., asymmetric gene flow between sub-Saharan Africa and Mediterranean populations).

The $f_{4}$ statistic is usually motivated in the context of a particular population model of randomly mating populations that may split or merge with each other (so their history is described by a simple admixture graph). Is it still useful even if history is not well-described as occasional interactions between a few randomly mating populations? A thought experiment is useful at this point. Suppose that in addition to nucleotide sequences, we also had access to any sort of genealogical information we might want. Would the genomes still be useful in fitting the model? The answer is no - since the model is neutral, genomes only provide information about past history insofar as they shed light on past genealogical relationships. In other words, the genealogies, if we had them, would be a sufficient statistic for inference of these population models. So, the $f_{4}$ statistic describes something about those genealogies that is informative about the presence or absence of gene flow between random mating populations. For understanding the $f_{4}$ statistic - both under the model above and in other contexts - it is helpful to describe this "something about those genealogies". Imagine annotating every possible ancestral individual (i.e., everyone who ever lived) with how much each set of samples inherit from that individual. The results below provide a (model-free) interpretation of the $f_{4}$ statistic as an estimator of a summary statistic of these annotations (illustrated in Figure 1).

Theorem 1 ( $f_{4}$ statistic). Let $X, Y, U$, and $V$ be collections of $n_{X}, n_{Y}, n_{U}$, and $n_{V}$ chromosomes, respectively, that have been genotyped at $G$ sites. Under the infinite-sites model of mutation, the following equivalences hold. (I) For each position $\ell$ in the genome and each ancestral individual's chromosome, a, let $F_{X}(a, \ell)$ denote the proportion of the samples in $X$ that have inherited from a at position $\ell$, and similarly for $F_{Y}, F_{U}$, and $F_{V}$. Then

$$
\mathbb{E}\left[f_{4}(X, Y ; U, V)\right]=\frac{1}{G} \sum_{\ell=1}^{G} \sum_{a} \mu(\ell)\left(F_{X}(a, \ell)-F_{Y}(a, \ell)\right)\left(F_{U}(a, \ell)-F_{V}(a, \ell)\right),
$$

where $\mu(\ell)$ is the per-generation mutation rate at site $\ell$, and the sum over $a$ is the sum over all ancestral chromosomes. (II) Equivalently, for any set of samples $(x, y, u, v)$, let $S_{x u \mid y v}(\ell)$ denote the lengths of any branches in the tree at position $\ell$ between the most recent common ancestor of $x$ and $u$ and any ancestor of $y$ or $v$, or between the most recent common ancestor of $y$ and $v$ and any ancestor of $x$ or $u$. (This is the length of the internal branch in the unrooted tree at position $\ell$ if it has topology $x u \mid y v$, and is zero otherwise.) Then

$$
\mathbb{E}\left[f_{4}(X, Y ; U, V)\right]=\frac{1}{n_{X} n_{Y} n_{U} n_{V}} \sum_{x, y, u, v} \frac{1}{G} \sum_{\ell=1}^{G} \mu(\ell)\left(S_{x u \mid y v}(\ell)-S_{x v \mid y u}(\ell)\right),
$$

where the sum is over all choices of $x \in X, y \in Y, u \in U$, and $v \in V$. Furthermore,

$$
\operatorname{var}\left[f_{4}(X, Y ; U, V)\right] \leq \frac{1}{n_{X} n_{Y} n_{U} n_{V}} \sum_{x, y, u, v} \frac{1}{G^{2}} \sum_{\ell=1}^{G} \mu(\ell)\left(S_{x u \mid y v}(\ell)+S_{x v \mid y u}(\ell)\right) .
$$

Note that $F_{X}(a, \ell)$ is unknown but quantifies the genetic inheritance of $X$, and so equation (2) interprets $f_{4}(X, Y ; U, V)$ as a descriptive statistic of demographic history. Once formulated using the general-purpose 

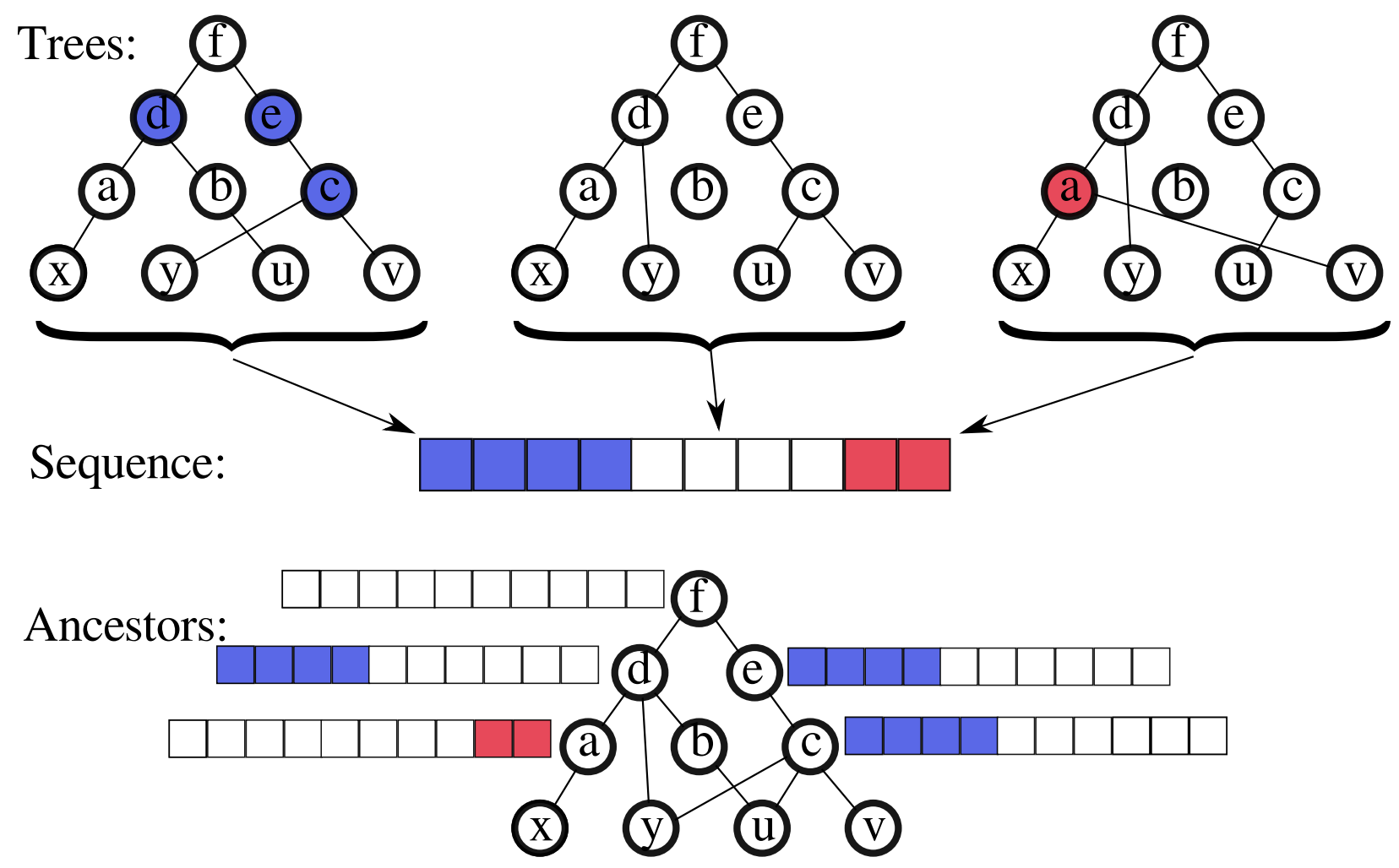

Figure 1: Depiction of calculating expected $f_{4}(x, y ; u, v)$ from the pedigree relating the four chromosomes $x, y, u$, and $v$. Top shows the three distinct trees found along the chromosome, and middle shows the locations on the 10-base-pair chromosome where those trees are found. Nodes in the trees are colored by their expected contribution to $f_{4}$ - any mutation occurring in blue nodes at that position will contribute $+\mu / 10$ to $f_{4}\left(x, y ; u, v 0\right.$, while mutations occurring in red nodes would contribute $-\mu / 10$, so that $\mathbb{E}\left[f_{4}(x, y ; u, v)\right]=$ $\mu(4 \times 3+0-2 \times 1)=10 \mu$. Bottom shows another way of computing this: individuals are provided with chromosomes labeled according to how they contribute to $f_{4}(x, y ; u, v)$. Instead of summing over trees, we can sum over ancestors, so that so that $\mathbb{E}\left[f_{4}(x, y ; u, v)\right]=\mu(4+4+4-2)=10 \mu$. 
theory, the proof of this comes down to straightforward calculations with Poisson processes, which are carried out below.

As a final example, the theory can be used to design a statistic, to estimate the covariance in times to most recent common ancestor between two pairs of chromosomes. This statistic has not to my knowledge been described in the literature. Concretely, let $\left(x_{1}, x_{2}\right)$ and $\left(y_{1}, y_{2}\right)$ be two pairs of sampled chromosomes, and for each position $\ell$ in the genome, let $t_{x}(\ell)$ denote the total number of ancestors from whom either $x_{1}$ or $x_{2}$, but not both, have inherited at $\ell$, and likewise for $t_{y}(\ell)$. (So, $t_{x}(\ell)$ is the length of the tree, in number of meioses, between $x_{1}$ and $x_{2}$ at $\ell$.) Suppose the genome is divided into nonoverlapping pieces $\left\{W_{1}, \ldots, W_{n}\right\}$, and we wish to estimate covariances of mean values of $t_{x}$ and $t_{y}$, scaled by mutation rate, across these chunks. (The scaling by mutation rate could be removed if it was constant across sites, or known, but I am leaving it in for realism.) In other words, we want the covariance between $\overline{\mu t}_{k, x}=\frac{1}{\left|W_{k}\right|} \sum_{\ell \in W_{k}} \mu_{\ell} t_{x}(\ell)$, where $\mu_{\ell}$ is the mutation rate at site $\ell$, and the corresponding quantity for $y$. For convenience, also let $\overline{\overline{\mu t}}_{x}=(1 / G) \sum_{\ell=1}^{G} \mu_{\ell} t_{x}(\ell)$ denote the mean time to common ancestor across the entire genome, scaled by mutation rate; and likewise for $\overline{\mu t}_{k, y}$ and $\overline{\overline{\mu t}}_{y}$.

Theorem 2 (Empirical covariance of mean coalescence times). Let $N_{k}(x)$ denote the number of mutations that differentiate $x_{1}$ and $x_{2}$ in window $W_{k}, N_{k}(y)$ the same for $y_{1}$ and $y_{2}$, and let $N_{k}(x, y)$ denote the number of mutations that do both, i.e., the number of mutations that differentiate between $x_{1}$ and $x_{2}$ as well as between $y_{1}$ and $y_{2}$. Define $Z_{j k}=N_{j}(x) N_{k}(y) /\left|W_{j}\right|\left|W_{k}\right|$ for $j \neq k$, and $Z_{j j}=\left(N_{j}(x) N_{j}(y)-N_{j}(x, y)\right) /\left|W_{j}\right|^{2}$. The statistic

$$
C_{x, y}:=\frac{1}{n} \sum_{k=1}^{n}\left(Z_{k k}-\frac{1}{n} \sum_{j=1}^{n} Z_{j k}\right)
$$

estimates the covariance in mean mutation-scaled coalescent times: under the infinite-sites model of mutation,

$$
\mathbb{E}\left[C_{x, y}\right]=\frac{1}{n} \sum_{k=1}^{n}\left(\overline{\mu t}_{k, x}-\overline{\overline{\mu t}}_{x}\right)\left(\overline{\mu t}_{k, y}-\overline{\overline{\mu t}}_{y}\right),
$$

with variance

$$
\operatorname{var}\left[C_{x, y}\right] \leq \frac{\epsilon^{3}}{n|W|}
$$

if $\epsilon=\max _{k}\left\{\max \left(\overline{\mu t}_{k, x}, \overline{\mu t}_{k, y}\right)\right\}$ and $|W|=\max _{k}\left|W_{k}\right|$.

Now, I use the theory of Poisson processes to generalize these examples.

\section{A formal calculus of mutations on the ARG}

Genealogy Ambitiously, the object of study is the collection of genetic relationships between every individual ever in the population, past and present. To simplify the discussion of relationships between diploids, consider relationships on only a single chromosome, and let $\mathcal{A}$ denote the collection of all copies of this chromosome in any individual alive at any point back to some arbitrarily distant time. The population pedigree, denoted $\mathcal{P}$, is the directed acyclic graph with vertex set $\mathcal{A}$ that has an edge $a \rightarrow b$ if $a$ is a parent of $b$, so that each vertex in $\mathcal{A}$ has in-degree equal to two. (Although this is a different object than the pedigree relating the diploid individuals, rather than their constituent chromosomes, the two are simple transformations of each other.) Under the standing assumption of the infinite sites model, no two mutations fall in the same location, and so locations on the chromosome are indexed by the continuous interval $\mathcal{G}=[0, G]$, where $G$ is the total length of the chromosome. At each site in $\mathcal{G}$ all ancestral chromosomes are related by a tree, embedded in the population pedigree, and this collection of trees is equivalent to the ARG. 
Mutation Under the infinite sites model, the mutation process is Poisson: concretely, the sites at which ancestral chromosome $a \in \mathcal{A}$ differs from their parent (i.e., has a mutation) are distributed as the points of an inhomogeneous Poisson process on $\mathcal{G}$. This Poisson process of mutations has intensity measure given by the function $\mu$, where $\mu(\ell)$ is the mutation rate at $\ell$. To study the inheritance of mutations through the pedigree, these are next collected into a Poisson process on all ancestral chromosomes: formally, $M$ is the point measure on $\mathcal{A} \times \mathcal{G}$ formed by putting one point mass at each mutation. Equivalently, $M$ counts mutations: for any region of the chromosome $[i, j] \subset \mathcal{G}$ and any set of ancestral chromosomes $A \subset \mathcal{A}$, the value $M(A \times[i, j])$ is the total number of mutations that occurred in the region $[i, j]$ on any of the chromosomes in $A$.

Under the assumption that mutation occurs independently of the pedigree, the expected number of mutations occurring in any collection of pieces of ancestral chromosomes is simply the sum of $\mu(\ell)$ across these pieces. Formally, $M$ 's mean measure has density $\mu$ with respect to $n \otimes d \ell$, where $n$ is counting measure on $\mathcal{A}$ and $d \ell$ is Lesbegue measure on the chromosome. Below, I will abuse notation slightly and write $d \mu$ for this measure, so that for any (measurable) subset of ancestral genomes $S \subset \mathcal{A} \times \mathcal{G}$,

$$
\mathbb{E}[M(S)]=\int_{S} d \mu(a, \ell) .
$$

The key simplifying assumption of the infinite sites model is that every mutation is observable: if two ancestral chromosomes $a$ and $b$ have both inherited a region of genome $[i, j]$ from their most recent common ancestor on this region, then given the genomes of $a$ and $b$ on this segment, we also know the number of mutations that have occurred in this segment in any of the ancestors going back to the common ancestor.

Additive statistics The formulation of mutations as a point measure on ancestral genomes is designed to make a certain set of statistics easy to formulate and analyze, namely, those that can be formed using integrals of test functions against the mutation process. Recall that since $M$ is a point measure on the set of all ancestral genomes, $\mathcal{A} \times \mathcal{G}$, with unit mass at each ancestral mutation, then if $\chi$ is a function on $\mathcal{A} \times \mathcal{G}$, the integral of $\chi$ against $M$ is the sum of the values of $\chi$ at the locations of all mutations: defining $\left\{m_{a, k}: 1 \leq k \leq n_{a}\right\}$ to be the locations of the mutations that occurred on ancestral chromosome $a$,

$$
\int \chi(a, \ell) d M(a, \ell)=\sum_{a \in \mathcal{A}} \sum_{k=1}^{n_{a}} \chi\left(a, m_{a, k}\right)
$$

It is easier to write this as simply $\int \chi d M$. Here is a simple example (for which all this notation is unnecessary):

Example 1 (Number of mutations). Given a sample of chromosomes $A \subset \mathcal{A}$ let $\zeta_{A}(a, \ell)=1$ if $a \in A$ and $\zeta_{A}(a, \ell)=0$ otherwise. Then $\int \zeta_{A} d M$ counts the number of mutations appearing de novo in the sampled chromosomes.

This is a simple prototype for what we would like to do more generally. But, notice that $\int \zeta_{A} d M$ is unobservable given only the genomes in $A$ - to identify new mutations, we need the parental genomes as well. Statistics of this form work by first, based on the samples, constructing a function $\chi$ on ancestral genomes, and then adding up the value that $\chi$ takes at the location of each mutation on any ancestral genome. To be observable, such statistics will need to satisfy certain conditions: in particular, $\chi$ must be zero for any piece of ancestral genome not inherited by any sampled genome, or inherited by all of them. This idea of observability under the infinite sites model is encoded by the following set of special functions:

Definition 1 (Paths). (I) For any two chromosomes $x$ and $y$ in $\mathcal{A}$, the path from $x$ to $y$ is the function

$$
[x \leftrightarrow y](a, \ell)= \begin{cases}1 & \text { if } x \text { or } y, \text { but not both, inherits from a at } \ell \\ 0 & \text { otherwise. }\end{cases}
$$



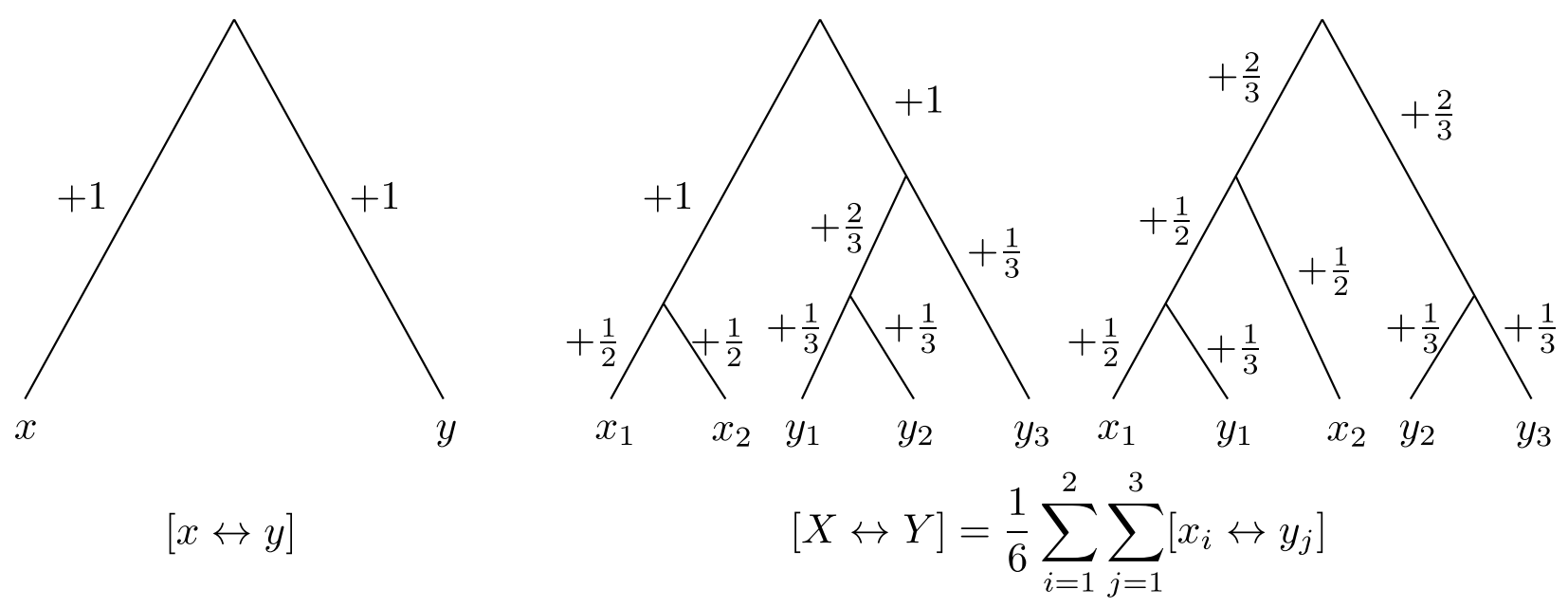

Figure 2: A cartoon of values taken by the path between (left) two sampled chromosomes $x$ and $y$, and (right) two groups of samples, $X=\left\{x_{1}, x_{2}\right\}$, and $Y=\left\{y_{1}, y_{2}, y_{3}\right\}$. The path between two single chromosomes is, marginally at each locus, the indicator of the path from each back to their common ancestor; but between larger groups the values it takes depends on the local topology of the tree relating the samples.

(II) For any two collections of chromosomes $X$ and $Y$, having $|X|$ and $|Y|$ elements each, the path from $X$ to $Y$ is the function

$$
[X \leftrightarrow Y](a, \ell)=\frac{1}{C(X, Y)} \sum_{x, y}[x \leftrightarrow y](a, \ell),
$$

where the sum is over distinct pairs $x \in X$ and $y \in Y$ with $x \neq y$, and $C(X, Y)$ is the number of such pairs. These are depicted in figure 2.

In other words, the path $[x \leftrightarrow y]$ is the indicator function of the pieces of ancestral chromosomes that are inherited by $x$ or $y$, but not both. At each locus $\ell$, the function $[x \leftrightarrow y](\cdot, \ell)$ on $\mathcal{A}$ is the indicator function of the tree connecting $x$ and $y$ at $\ell$ (but notably excluding the root). $[X \leftrightarrow Y]$ is the average of these indicators, which gives the proportion of paths between pairs of samples from the two groups that pass through each ancestor in the pedigree.

Example 2 (Sequence divergence). The sequence divergence between two chromosomes $x$ and $y$, denoted $\pi(x, y)$, is the mean density of distinguishing mutations; it is

$$
\pi(x, y)=\frac{1}{G} \int[x \leftrightarrow y] d M .
$$

For a group of samples $X$ we define the average pairwise divergence similarly:

$$
\begin{aligned}
\pi(X) & =\frac{1}{|X|(|X|-1)} \sum_{x_{1} \neq x_{2} \in X} \pi\left(x_{1}, x_{2}\right) \\
& =\frac{1}{G} \int([X \leftrightarrow X]) d M
\end{aligned}
$$

Tajima [1983] proposed $\pi(X)$ as an estimator for $\theta=4 N_{e} \mu$, under the model of a large, randomly mating population of diploids with constant mutation rate $\mu$ and effective population size $N_{e}$. Indeed, $\mathbb{E}[\pi(X)]=\theta$, 
but the variance of $\pi(X)$ for a nonrecombining locus does not go to zero as the number of samples grows, and so this is regarded as a poor estimator of $\theta$. However, it is clear that the variance does go to zero if calculated with the gene tree fixed, i.e., thought of as an estimator of the mean time to most recent common ancestor (multiplied by twice the mutation rate). Denoting by $\overline{\mu t}$ the empirical mean mutation-rate-scaled time to most recent common ancestor, the variance can be partitioned (as in Edwards and Beerli [2000]): $\operatorname{var}[\pi(X)]=\mathbb{E}[\operatorname{var}[\pi(X) \mid \overline{\mu t}]]+\operatorname{var}[\mathbb{E}[\pi(X) \mid \overline{\mu t}]]$. The first term here goes to zero as the size of the sample increases, but the second, due to randomness in the demographic process, does not. The question of whether $\pi(X)$ is a good estimator, and for what, becomes a question of whether we want to think of it as estimating a summary statistic of actual relationships or a parameter in a certain stochastic model of demography.

Observable statistics For application to data, it only makes sense to consider statistics that can be calculated using a sampled set of genomes. As discussed above, under the infinite sites model this means that the genome sequence of a pair of samples $x$ and $y$ on subset of the genome $L$ is determined by the number of mutations inherited by $x$ or $y$ but not both on $L$, or $\sum_{a \in \mathcal{A}} \sum_{\ell \in L}[x \leftrightarrow y](a, \ell)$. If $\mathbf{1}_{L}(a, \ell)=1$ for $\ell \in L$ and is zero otherwise, this is $M\left(\mathbf{1}_{L}[x \leftrightarrow y]\right)$, so the set of observable statistics, given a collection of samples $A$, is generated by the set of linear combinations of functions of this form, i.e., products of indicators of a segment of genome with a path between two samples. It is clear that we cannot learn about parts of the pedigree from which no samples have inherited, or about anything occurring longer ago than the most recent common ancestor of the samples at each site, but it is not clear how to formulate more generally what information about the ARG is or is not obtainable from finite samples of chromosomes.

This notion of observability is made formal by the following definition:

Definition 2 (Observable statistics). A function $\chi(a, \ell)$ on $\mathcal{A} \times \mathcal{G}$ is observable given a collection of chromosomes $A$ if it is in the algebra generated by functions of the form $\mathbf{1}_{[u, v]}(a, \ell)[x \leftrightarrow y](a, \ell)$, where $[u, v]$ is an interval of the chromosome, and $x$ and $y$ are samples in $A$.

There are further, unavoidable, limitations to what it is possible to learn from the data. For instance, the sequence divergence $\pi(x, y)$ between $x$ and $y$ is an estimate of the mean of the empirical distribution of times to most recent common ancestors (TMRCA) between $x$ and $y$, multiplied by the mutation rate. Theorem 2 gives an estimator of the variance of the mean TMRCA in windows. However, it is not possible to estimate the variance of the distribution of single-site TMRCA values without somehow modeling dependencies between sites induced by recombination. An easy way to see this is if each site has at most one mutation, then without using inter-site dependencies, the model is equivalent to one where the probability of a segregating mutation is constant, equal to the mean. To make further progress, it is necessary (and reasonable) to make further assumptions, that are beyond the scope of this paper.

\section{Moments}

Calculations with statistics in this formalism can be made with the help of the following general formula for integrals of test functions against a Poisson processes:

Lemma 1. [Generating function] For any test function $\phi: \mathcal{A} \times \mathcal{G} \rightarrow \mathbb{R}$ for which $\int \phi d \mu$ is absolutely convergent, the statistic $\int \phi d M$ is well-defined, and

$$
\mathbb{E}\left[\exp \left(\int \phi d M\right)\right]=\exp \left(\int\left(e^{\phi}-1\right) d \mu\right)
$$

Proof. This is Campbell's Theorem; see e.g., Kingman [1993]. Recall that $\int \phi d M$ is the sum of the value that $\phi$ takes over all mutations occurring in $M$; "well-defined" means that this sum is absolutely convergent even if there are an infinite number of mutations.

We immediately get, by differentiating this formula, the moments: 
Lemma 2 (Mean and covariance). Let $\phi$ and $\psi$ be test functions on $\mathcal{A} \times \mathcal{G}$ for which $\int \phi d \mu, \int \psi d \mu$, and $\int \phi \psi d \mu$ are all absolutely convergent. Then

$$
\begin{aligned}
\mathbb{E}\left[\int \phi d M\right] & =\int \phi d \mu \\
\operatorname{cov}\left[\int \phi d M, \int \psi d M\right] & =\int \phi \psi d \mu .
\end{aligned}
$$

Proof. These are a standard calculations, but we carry out the second for covariance for concreteness. Let $F(a, b)=\exp \left(\int(a \phi+b \psi) d M\right)$. Using (15),

$$
\left.\partial_{a} \partial_{b} \log \mathbb{E}[F(a, b)]\right|_{a=b=0}=\int \phi \psi d \mu
$$

On the other hand, exchanging the expectation and the derivatives,

$$
\begin{aligned}
\left.\partial_{a} \partial_{b} \log \mathbb{E}[F(a, b)]\right|_{a=b=0} & =\left.\frac{\mathbb{E}\left[\partial_{a} \partial_{b} F(a, b)\right]-\mathbb{E}\left[\partial_{a} F(a, b)\right] \mathbb{E}\left[\partial_{b} F(a, b)\right]}{\mathbb{E}\left[F(a, b)^{2}\right]}\right|_{a=b=0} \\
& =\mathbb{E}\left[\int \phi \psi d M\right]-\mathbb{E}\left[\int \phi d M\right] \mathbb{E}\left[\int \psi d M\right]
\end{aligned}
$$

Expressions for more general moments, or statistics whose expected values match desired quantities, can be found using the following general formula:

Lemma 3. [General Moments] Let $\mathcal{S}$ be the set of upper triangular $n \times n$ matrices with entries in $\{0,1\}$ and whose columns sum to 1 (i.e., ways of partitioning the set $\{1,2, \ldots, n\}$ into sets), and for $\sigma \in \mathcal{S}$ let $|\sigma|$ denote the number of nonzero rows of $\sigma$ (i.e., the number of sets in the partition). Then for any collection of test functions $\phi_{1}, \ldots, \phi_{n}$ on $\mathcal{A} \times \mathcal{G}$ for which the following integrals against $\mu$ are absolutely convergent,

$$
\mathbb{E}\left[\prod_{i=1}^{n} \int \phi_{i} d M\right]=\sum_{\sigma \in \mathcal{S}} \prod_{i} \int\left(\prod_{j} \phi_{j}^{\sigma_{i j}}\right) d \mu
$$

and

$$
\int \prod_{i=1}^{n} \phi_{i} d \mu=\sum_{\sigma \in \mathcal{S}}(-1)^{1+|\sigma|} \mathbb{E}\left[\prod_{i} \int\left(\prod_{j} \phi_{j}^{\sigma_{i j}}\right) d M\right] .
$$

Note: methods for efficient computation of mixed moments using orthogonal polynomials are presented in a general framework by Peccati and Taqqu [2011].

Proof. By induction, by differentiating either the generating function (for the first formula) or the log of the generating function (for the second) of $\sum_{i} \alpha_{i} \phi_{i}$.

\section{Application of the method}

We can now use the formalism developed above to easily prove the theorems given in the Introduction.

Proof of Theorem 1. Write $G_{x \ell}$ for the genotype of haplotype $x$ at position $\ell$, coded as '0' for the reference allele and ' 1 ' for the alternate allele. First note that

$$
f_{4}(X, Y ; U, V)=\frac{1}{G} \sum_{\ell=1}^{G} \frac{1}{n_{X} n_{Y} n_{U} n_{V}} \sum_{x, y, u, v}\left(G_{x \ell}-G_{y \ell}\right)\left(G_{u \ell}-G_{v \ell}\right),
$$


i.e., $f_{4}$ is the average product of differences between pairs of haplotypes chosen from $(X, Y)$ and from $(U, V)$, averaging over site in the genome and choices of pairs $(x, y)$ and $(u, v)$. For a given quadruple $(x, y, u, v)$, sites will add to this sum if there is a mutation shared by $x$ and $u$ that $y$ and $v$ do not carry, or vice versa; and sites will subtract from the sum if there is a mutation that is shared by $x$ and $v$ that $y$ and $u$ do not carry, or vice versa. The theorem for $f_{4}(x, y ; u, v)$ then follows easily by writing the statistic as the difference of two Poisson random variables, divided by $G$. To carry this through using the notation here, note that the function $[x \leftrightarrow v][y \leftrightarrow u]-[x \leftrightarrow u][y \leftrightarrow v]$ takes the value +1 on the internal branch of trees with unrooted topology $((x, u),(y, v)),-1$ on the internal branch of trees with unrooted topology $((x, v),(y, u))$, and is zero on the remaining topology $((x, y),(u, v))$; therefore,

$$
f_{4}(X, Y ; U, V)=\frac{1}{G} M([X \leftrightarrow V][Y \leftrightarrow U]-[X \leftrightarrow U][Y \leftrightarrow V]) .
$$

The expected value of $f_{4}$ is therefore just the integral of $[X \leftrightarrow V][Y \leftrightarrow U]-[X \leftrightarrow U][Y \leftrightarrow V]$ against $\mu$; using linearity this can be rewritten in various ways to give the two interpretations given in the theorem. Since $S_{x u \mid y v}(\ell)$ is equal to the length of the internal branch of the tree at $\ell$ if it has unrooted topology $((x, u),(y, v))$ and is zero otherwise,

$$
\mu\left(\mathbf{1}_{\{\ell\}}[x \leftrightarrow v][y \leftrightarrow u]-[x \leftrightarrow u][\leftrightarrow y] v\right)=\mu(\ell)\left(S_{x u \mid y v}(\ell)-S_{x v \mid y u}(\ell)\right),
$$

and so expression (3) follows immediately. (An alternative path would be to use the four point condition [Semple and Steel, 2003], which implies here that $[x \leftrightarrow v][y \leftrightarrow u]-[x \leftrightarrow u][y \leftrightarrow v]=([x \leftrightarrow v]+[y \leftrightarrow u]-$ $[x \leftrightarrow u]-[y \leftrightarrow v]) / 2$.)

For the first interpretation, define $\star$ to be all ancestral genomes alive at some fixed point in the remote past (longer ago than the maximum time to most recent common ancestor across the genome), and note that $[x \leftrightarrow u]=|\star|[\star \leftrightarrow x]+|\star|[\star \leftrightarrow u]-2|\star|^{2}[\star \leftrightarrow x][\star \leftrightarrow u]$. (The factors of $|\star|$ are only to cancel the denominator in the definition of the path function.) Therefore, $[x \leftrightarrow v][y \leftrightarrow u]-[x \leftrightarrow u][y \leftrightarrow v]=$ $|\star|^{2}([\star \leftrightarrow x]-[\star \leftrightarrow y])([\star \leftrightarrow u]-[\star \leftrightarrow v])$. Since $\mu(|\star|[\star \leftrightarrow x])(a, \ell)$ is equal to 1 if $a$ is more recent than the age of the ancestors in $\star$ and $x$ has inherited from $a$ at $\ell$, averaging over choices of $x$ gives the function $F_{X}$ defined in the theorem: $|\star| \mu([\star \leftrightarrow X])(a, \ell)=\mu(\ell) F_{X}(a, \ell)$, so that equation (2) follows. Note that in this interpretation, each term in the product is not summable on its own (e.g., $\sum_{a} F_{X}(a, \ell)=\infty$ ), but cancellation and a reasonable assumption about finiteness of recent common ancestors makes differences like $F_{X}-F_{Y}$ summable.

Finally, consider the variance. By Lemma 2,

$$
\operatorname{var}\left[f_{4}(X, Y ; U, V)\right]=\frac{1}{G^{2}} \mu\left(\{[X \leftrightarrow V][Y \leftrightarrow U]-[X \leftrightarrow U][Y \leftrightarrow V]\}^{2}\right) .
$$

By Jensen's inequality,

$$
([X \leftrightarrow V][Y \leftrightarrow U]-[X \leftrightarrow U][Y \leftrightarrow V])^{2} \leq \frac{1}{n_{X} n_{Y} n_{U} n_{V}} \sum_{x, y, u, v}([x \leftrightarrow v][y \leftrightarrow u]-[x \leftrightarrow u][y \leftrightarrow v])^{2} .
$$

Since the summand is an indicator function,

$$
\mu\left(\mathbf{1}_{\{\ell\}}([x \leftrightarrow v][y \leftrightarrow u]-[x \leftrightarrow u][y \leftrightarrow v])^{2}\right)=S_{x u \mid y v}(\ell)+S_{x v \mid y u}(\ell) .
$$

Summed over $\ell$, this gives equation (4).

Theorem 2 describes how to estimate the covariance in empirical mean coalescent times between two pairs of sampled chromosomes. Since the probability that two samples are heterozygous (i.e., differ) at a site is proportional to their TMRCA at that site (to first order), it is natural to suggest the covariance of heterozygosities as an estimator of at least a similar quantity. This would be arguably more natural from the 
population genetics point of view, as mean heterozygosities are an easily observed statistic. The following is therefore a useful contrast to theorem 2 .

First, some more notation. Let $\left(x_{1}, x_{2}\right)$ and $\left(y_{1}, y_{2}\right)$ be two pairs of sampled chromosomes, and for each position $\ell$ in the genome, recall that $t_{x}(\ell)$ is the total number of ancestors in the tree leading from $x_{1}$ and $x_{2}$ back to their common ancestor at $\ell$ (not including the common ancestor), and likewise for $t_{y}(\ell)$. We will also need $t_{x \cap y}(\ell)$, the number of ancestors in the intersection of these two trees. Just as $\overline{\mu t}_{x}$ was defined above to be the mean time to common ancestor, scaled by mutation rate; so also $\overline{\mu t}_{x \cap y}=(1 / G) \sum_{\ell=1}^{G} \mu_{\ell} t_{x \cap y}(\ell)$.

Theorem 3 (Empirical covariance of heterozygosities). Let $\theta_{x}(\ell)=1$ if the genotypes of $x_{1}$ and $x_{2}$ differ at $\ell$, and $\theta_{x}(\ell)=0$ otherwise, and let $\bar{\theta}_{x}=(1 / G) \sum_{\ell=1}^{G} \theta_{x}(\ell)$ be the mean heterozygosity of $\left(x_{1}, x_{2}\right)$; and likewise for $\theta_{y}(\ell)$ and $\bar{\theta}_{y}$. Then, under the infinite-sites model of mutation,

$$
\mathbb{E}\left[\left(\frac{G-1}{G^{2}} \sum_{\ell=1}^{G} \theta_{x}(\ell) \theta_{y}(\ell)\right)-\bar{\theta}_{x} \bar{\theta}_{y}\right]=\overline{\mu t}_{x \cap y}-\overline{\mu t}_{x} \overline{\mu t}_{y}
$$

and

$$
\operatorname{var}\left[\left(\frac{G-1}{G^{2}} \sum_{\ell=1}^{G} \theta_{x}(\ell) \theta_{y}(\ell)\right)-\bar{\theta}_{x} \bar{\theta}_{y}\right]=\frac{1}{G} t_{x \cap y}(\ell)+O\left(1 / G^{3}\right) .
$$

Proof of Theorem 3. Under the infinite-sites model, $\theta_{x}(\ell)$ and $\theta_{y}(\ell)$ are both nonzero only if there is a mutation at $\ell$ that falls on both the path from $x_{1}$ to $x_{2}$ and the path from $y_{1}$ to $y_{2}$, and so

$$
\sum_{\ell} \theta_{x}(\ell) \theta_{y}(\ell)=\int\left[x_{1} \leftrightarrow x_{2}\right](a, \ell)\left[y_{1} \leftrightarrow y_{2}\right](a, \ell) d M(a, \ell) .
$$

For convenience, let $\phi_{x}=\left[x_{1} \leftrightarrow x_{2}\right], \phi_{y}=\left[y_{1} \leftrightarrow y_{2}\right]$, and for a test function $\psi$ write $M(\psi)=\int \psi d M$ and $\mu(\psi)=\int \psi d \mu$. In this shorthand, the statistic is

$$
\left(\frac{G-1}{G^{2}} \sum_{\ell=1}^{G} \theta_{x}(\ell) \theta_{y}(\ell)\right)-\bar{\theta}_{x} \bar{\theta}_{y}=\frac{G-1}{G^{2}} M\left(\phi_{x} \phi_{y}\right)-\frac{1}{G^{2}} M\left(\phi_{x}\right) M\left(\phi_{y}\right) .
$$

Using Lemma $3, \mathbb{E}\left[M\left(\phi_{x} \phi_{y}\right)\right]=\mu\left(\phi_{x} \phi_{y}\right)$ and $\mathbb{E}\left[M\left(\phi_{x}\right) M\left(\phi_{y}\right)\right]=\mu\left(\phi_{x} \phi_{y}\right)+\mu\left(\phi_{x}\right) \mu\left(\phi_{y}\right)$, which combine to give

$$
\mathbb{E}\left[\frac{G-1}{G^{2}} M\left(\phi_{x} \phi_{y}\right)-\frac{1}{G^{2}} M\left(\phi_{x}\right) M\left(\phi_{y}\right)\right]=\frac{1}{G} \mu\left(\phi_{x} \phi_{y}\right)-\frac{1}{G^{2}} \mu\left(\phi_{x}\right) \mu\left(\phi_{y}\right),
$$

which is equation (29).

Again using Lemma 3, and the fact that since the value of $\phi_{x}(a, \ell)$ is everywhere either 0 or 1 , so $\phi_{x}(a, \ell)^{2}=\phi_{x}(a, \ell), \operatorname{var}\left[M\left(\phi_{x} \phi_{y}\right)\right]=\mu\left(\phi_{x} \phi_{y}\right)$ and

$$
\operatorname{cov}\left[M\left(\phi_{x} \phi_{y}\right), M\left(\phi_{x}\right) M\left(\phi_{y}\right)\right]=\mu\left(\phi_{x} \phi_{y}\right)\left(1+\mu\left(\phi_{x}\right)+\mu\left(\phi_{y}\right)\right)
$$

and

$$
\operatorname{var}\left[M\left(\phi_{x}\right) M\left(\phi_{y}\right)\right]=\mu\left(\phi_{x} \phi_{y}\right)\left(1+2\left(\mu\left(\phi_{x}\right)+\mu\left(\phi_{y}\right)\right)+2 \mu\left(\phi_{x} \phi_{y}\right)+4 \mu\left(\phi_{x}\right) \mu\left(\phi_{y}\right)\right)
$$

so finally, equation (30) is $1 / G^{4}$ multiplied by

$$
\begin{gathered}
(G-1)^{2} \operatorname{var}\left[M\left(\phi_{x} \phi_{y}\right)\right]-2(G-1) \operatorname{cov}\left[M\left(\phi_{x} \phi_{y}\right), M\left(\phi_{x}\right) M\left(\phi_{y}\right)\right]+\operatorname{var}\left[M\left(\phi_{x}\right) M\left(\phi_{y}\right)\right] \\
=\mu\left(\phi_{x} \phi_{y}\right)\left\{(G-1)^{2}-2(G-1)\left(1+\mu\left(\phi_{x}\right)+\mu\left(\phi_{y}\right)\right)\right. \\
\left.+1+2\left(\mu\left(\phi_{x}\right)+\mu\left(\phi_{y}\right)\right)+2 \mu\left(\phi_{x} \phi_{y}\right)+4 \mu\left(\phi_{x}\right) \mu\left(\phi_{y}\right)\right\} .
\end{gathered}
$$


We now turn to the proof of Theorem 2, which is similar to the previous proof, but somewhat more involved.

Proof of Theorem 2. Here, the goal is to design a statistic that estimates the covariance in mean coalescent times. We scale time by mutation rate because numbers of mutations only depend on the product of branch length and mutation rate, which therefore cannot be disentangled without additional assumptions (or modeling of recombination.) Therefore, if we define

$$
\psi_{k, x}(a, \ell)= \begin{cases}\frac{1}{\mid W_{k}}\left[x_{1} \leftrightarrow x_{2}\right](a, \ell) & \text { if } \ell \in W_{k} \\ 0 & \text { otherwise }\end{cases}
$$

then $\mu\left(\psi_{k, x}\right)=\overline{\mu t}_{k, x}$ gives the mean coalescent time on window $W_{k}$. We next need something whose expectation is $\mu\left(\psi_{k, x}\right) \mu\left(\psi_{k, y}\right)$. By Lemma 3 , this is

$$
\mathbb{E}\left[M\left(\psi_{k, x}\right) M\left(\psi_{k, y}\right)-M\left(\psi_{k, x} \psi_{k, y}\right)\right]=\mu\left(\psi_{k, x}\right) \mu\left(\psi_{k, y}\right) .
$$

Similarly, if we define $\bar{\psi}_{x}(a, \ell)=\frac{1}{n} \sum_{k=1}^{n} \psi_{k, x}(a, \ell)$, so that $\mu\left(\bar{\psi}_{x}\right)=\overline{\overline{\mu t}}_{x}$, we need something whose expectation is $\mu\left(\bar{\psi}_{x}\right) \mu\left(\bar{\psi}_{y}\right)$, which by the same lemma is

$$
\mathbb{E}\left[M\left(\bar{\psi}_{x}\right) M\left(\bar{\psi}_{y}\right)-M\left(\bar{\psi}_{x} \bar{\psi}_{y}\right)\right]=\mu\left(\bar{\psi}_{x}\right) \mu\left(\bar{\psi}_{y}\right) .
$$

These two combine to get

$$
C_{x, y}=\frac{1}{n} \sum_{k=1}^{n}\left\{M\left(\psi_{k, x}\right) M\left(\psi_{k, y}\right)-M\left(\psi_{k, x} \psi_{k, y}\right)\right\}-\left\{M\left(\bar{\psi}_{x}\right) M\left(\bar{\psi}_{y}\right)-M\left(\bar{\psi}_{x} \bar{\psi}_{y}\right)\right\} .
$$

Now note that by linearity of $M$,

$$
M\left(\bar{\psi}_{x}\right) M\left(\bar{\psi}_{y}\right)-M\left(\bar{\psi}_{x} \bar{\psi}_{y}\right)=\frac{1}{n^{2}} \sum_{j=1}^{n} \sum_{k=1}^{n}\left(M\left(\psi_{j, x}\right) M\left(\psi_{k, y}\right)-M\left(\psi_{j, x} \psi_{k, y}\right)\right) .
$$

Defining $Z_{j k}=M\left(\psi_{j, x}\right) M\left(\psi_{k, y}\right)-M\left(\psi_{j, x} \psi_{k, y}\right)$, then

$$
C_{x, y}=\frac{1}{n} \sum_{k=1}^{n} Z_{k k}-\frac{1}{n^{2}} \sum_{j=1}^{n} \sum_{k=1}^{n} Z_{j k}
$$

which, since $M\left(\psi_{k, x}\right)=N_{k}(x) /\left|W_{k}\right|$ and $M\left(\psi_{k, x} \psi_{k, y}\right)=N_{k}(x, y) /\left|W_{k}\right|^{2}$, is equation (5).

The substantially lengthier calculation of the variance is postponed until the Appendix.

\section{The infinite sites assumption}

Some of the simplicity above (and more generally in population genetics) relies on the assumption that only one mutation can occur at each site, which generally results in expressions that are correct up to a factor proportional to the fraction of sites at which more than one mutation has occurred. To illustrate this, suppose that more than one mutation can occur per site; in other words, the Poisson process of mutations happens on a discrete, not continuous, set.

Assume for the moment that all mutation rates are equal: $\mu_{\ell}=\mu$. Fix a pairs of sampled chromosomes $\left(x_{1}, x_{2}\right)$, and define $\xi_{n}(\ell)$ is defined to be 1 if $n$ mutations have occurred at site $\ell$ between $x_{1}$ and $x_{2}$, and let $\bar{\xi}_{n}=(1 / G) \sum_{\ell=1}^{G} \xi_{n}(\ell)$. The expected value of $\bar{\xi}_{n}$ under this model is

$$
\bar{G} \sum_{\ell=1}^{G} e^{-\mu t_{x}(\ell)} \frac{\left(\mu t_{x}(\ell)\right)^{n}}{n !}
$$


and so an estimate of $n^{\text {th }}$ moment of the empirical distribution of $t_{x}(\ell)$ could be $n ! \frac{\bar{\xi}_{n}}{\xi_{0}}$. However, since this is essentially estimating the density of sites at which there were $n$ mutations, to have any accuracy requires a reasonable number of such sites, and distinguishing these from sequencing error. In practice, this is highly problematic, and is confounded by mutation rate heterogeneity.

\section{Discussion}

In this paper I have explored an empirical approach to demographic inference in population genetics, by treating the (empirical, realized) ancestral recombination graph as a complex, unobserved, object we wish to learn about, rather than as an intermediate layer that is averaged over in the course of inferring parameters of interest in higher-level stochastic models (e.g., coalescent models). This approach certainly does not replace coalescent theory, but seems useful in that it can provide more concretely interpretable results to nonspecialists (e.g., "numbers of common ancestors" rather than "coalescent rates"), and intuition about what statistics have the best power to distinguish between alternative population models. This way of thinking is certainly not new, but data that give us power to infer quantities directly at this level of abstraction is.

Additionally, I have described a new formalism that can simplify calculations related to population genetics statistics by writing these as integrals of functions against a Poisson random measure, which models the locations of mutations on the genomes of all possible ancestors.

This point of view, and this formalism, leads to two general sorts of interpretations for a given statistic: first, in terms of the distribution of trees along which the samples are related at each locus; and second, in terms of weighted sums over ancestral chromosomes. This duality is easy to see considering simple examples such as pairwise divergences, and is less obvious for more complex statistics such as $f_{4}$.

$f_{4}$ and family Theorem 1 gives an interpretation of the $f_{4}$ statistic as a sum of products of differences in ancestry, over all ancestral genomes. Patterson et al. [2012] interprets this and related statistics in terms of shared drift along branches of an admixture graph (in which each edge is a randomly mating population). Since genetic drift is determined by the sharing of common ancestors, Theorem 1 can be seen as a more precise statement of the same observation. Since the main point of this paper is to lay out the general framework, I have not undertaken the task of recasting the entire family of related statistics (e.g., ABBA-BABA), but the general way forward can be seen by analogy to $f_{4}$.

The unknown network of ancestors The problem at hand, to infer aspects of the unknown pedigree through which genetic material has been noisily inherited, has some parallels to the problem of active network tomography [reviewed in Lawrence et al., 2006]: given information on losses and delays of "probe" packets sent between a subset of peripheral nodes in a network, infer the topology of the network and certain characteristics of its internal nodes. Results in this field on identifiability [e.g., Singhal and Michailidis, 2007, Gopalan and Ramasubramanian, 2012] would be very interesting in this context, although genomic data are substantially noisier, more sparsely collected, and more numerous. Others have already made use of the parallels to phylogenetics, in the other direction [Ni and Tatikonda, 2011].

Recombination A glaring omission in this paper is any treatment of linkage between sites: for instance, when calculating variances, each site is treated as independent; surely this cannot be right, as the trees at nearby sites are highly correlated? But, we begin by assuming that the mutation process at each site is independent given the trees (which seems for the most part reasonable), and take the entire empirical ancestral recombination graph as fixed, which includes the locations of ancestral recombination breakpoints. If the aim is to estimate descriptive statistics of the ARG then this is the correct point of view; but an intermediate position would be to treat only the population pedigree as given, and to additionally model randomness in recombination. This could allow for recovery of additional information left behind by recombination, and was the point of view taken in Ralph and Coop [2013]. This will be the subject of future work. 
Mutation Above, we have allowed for heterogeneity in mutation rate, as this is known to be substantial [e.g., Misawa and Kikuno, 2009]. When applying these methods, it seems necessary to choose regions of the genome with comparable large-scale mutation rates, and then within these, to average over enough sites that small-scale heterogeneity will not hopelessly confound comparisons. Methods such as described in Lipson et al. [2015], may be useful in disentangling mutation rate heterogeneity from heterogeneity in demographic histories along the genome. Furthermore, this paper only models diallelic markers, and completely ignores both sequence context and backmutation. This is common in population genetics methods, since the infinite sites assumption should be a good one at the typical levels of divergence encountered within species, especially if mutation rate heterogeneity in accounted for. Reconciliation of more realistic models of mutation with the Poisson process method described here would require more work.

Selection An important assumption behind this method is that mutations are independent of the ARG. This is not true for alleles under selection, but neither does this approach entirely assume neutrality: since the ARG is taken as given, if we knew which sites were under selection and excluded these from the analyses, then the assumptions would be satisfied. In other words, linked, selected sites are not an issue, as they act by distorting nearby gene trees. On the other hand, if a large fraction of segregating polymorphisms are actively under selection, this violates the model (but it is unclear what to replace it with).

\section{Acknowledgements}

Thanks to Paul Marjoram, Graham Coop, and Yaniv Brandvain for useful discussion, to John Wakeley for insightful comments, and to an anonymous reviewer for correcting an error in the proof Theorem 1 and otherwise greatly improving the manuscript. This work was supported by NSF grant \#1262645 (DBI) and startup funds from USC.

\section{References}

J C Avise, J Arnold, R M Ball, E Bermingham, T Lamb, J E Neigel, C A Reeb, and N C Saunders. Intraspecific phylogeography: The mitochondrial DNA bridge between population genetics and systematics. Annual Review of Ecology and Systematics, 18(1):489-522, 1987. doi: 10.1146/annurev.es.18.110187.002421. URL https://doi.org/10.1146/annurev.es.18.110187.002421.

Clare Bycroft, Colin Freeman, Desislava Petkova, Gavin Band, Lloyd T Elliott, Kevin Sharp, Allan Motyer, Damjan Vukcevic, Olivier Delaneau, Jared O'Connell, Adrian Cortes, Samantha Welsh, Gil McVean, Stephen Leslie, Peter Donnelly, and Jonathan Marchini. Genome-wide genetic data on 500,000 UK Biobank participants. bioRxiv, 2017. doi: 10.1101/166298. URL https://www.biorxiv.org/content/ early/2017/07/20/166298.

R L Cann, M Stoneking, and A C Wilson. Mitochondrial DNA and human evolution. Nature, 325(6099): 31-36, January 1987. doi: 10.1038/325031a0. URL http://www.ncbi.nlm.nih.gov/pubmed/3025745.

Peter de Barros Damgaard, Nina Marchi, Simon Rasmussen, Michal Peyrot, Gabriel Renaud, Thorfinn Korneliussen, J. Vctor Moreno-Mayar, Mikkel Winther Pedersen, Amy Goldberg, Emma Usmanova, Nurbol Baimukhanov, Valeriy Loman, Lotte Hedeager, Anders Gorm Pedersen, Kasper Nielsen, Gennady Afanasiev, Kunbolot Akmatov, Almaz Aldashev, Ashyk Alpaslan, Gabit Baimbetov, Vladimir I. Bazaliiskii, Arman Beisenov, Bazartseren Boldbaatar, Bazartseren Boldgiv, Choduraa Dorzhu, Sturla Ellingvag, Diimaajav Erdenebaatar, Rana Dajani, Evgeniy Dmitriev, Valeriy Evdokimov, Karin M. Frei, Andrey Gromov, Alexander Goryachev, Hakon Hakonarson, Tatyana Hegay, Zaruhi Khachatryan, Ruslan Khaskhanov, Egor Kitov, Alina Kolbina, Tabaldiev Kubatbek, Alexey Kukushkin, Igor Kukushkin, Nina Lau, Ashot Margaryan, Inga Merkyte, Ilya V. Mertz, Viktor K. Mertz, Enkhbayar Mijiddorj, Vyacheslav Moiyesev, Gulmira Mukhtarova, Bekmukhanbet Nurmukhanbetov, Z. Orozbekova, Irina Panyushkina, Karol Pieta, Vclav Smrka, Irina Shevnina, Andrey Logvin, Karl-Gran Sjgren, Tereza tolcov, Kadicha 
Tashbaeva, Alexander Tkachev, Turaly Tulegenov, Dmitriy Voyakin, Levon Yepiskoposyan, Sainbileg Undrakhbold, Victor Varfolomeev, Andrzej Weber, Nikolay Kradin, Morten E. Allentoft, Ludovic Orlando, Rasmus Nielsen, Martin Sikora, Evelyne Heyer, Kristian Kristiansen, and Eske Willerslev. 137 ancient human genomes from across the Eurasian steppes. Nature, 557(7705):369-374, 2018. ISSN 14764687. doi: 10.1038/s41586-018-0094-2. URL https://doi.org/10.1038/s41586-018-0094-2.

E Y Durand, N Patterson, D Reich, and M Slatkin. Testing for ancient admixture between closely related populations. Mol Biol Evol, 28(8):2239-2252, August 2011. doi: 10.1093/molbev/msr048. URL http: //www.ncbi.nlm.nih.gov/pubmed/21325092.

Scott V. Edwards and Peter Beerli. Perspective: gene divergence, population divergence, and the variance in coalescence time in phylogeographic studies. Evolution, 54(6):1839-1854, 2000. ISSN 1558-5646. doi: 10. 1111/j.0014-3820.2000.tb01231.x. URL http://dx.doi.org/10.1111/j.0014-3820.2000.tb01231.x.

W.J. Ewens. Mathematical population genetics. Springer, 2004.

John H. Gillespie and Charles H. Langley. Are evolutionary rates really variable? Journal of Molecular Evolution, 13(1):27-34, 1979. ISSN 0022-2844. doi: 10.1007/BF01732751. URL http://dx.doi.org/10. 1007/BF01732751.

Abishek Gopalan and Srinivasan Ramasubramanian. On identifying additive link metrics using linearly independent cycles and paths. IEEE/ACM Trans. Netw., 20(3):906-916, June 2012. ISSN 1063-6692. doi: 10.1109/TNET.2011.2174648. URL http://dx.doi.org/10.1109/TNET . 2011.2174648.

R E Green, J Krause, A W Briggs, T Maricic, U Stenzel, M Kircher, N Patterson, H Li, W Zhai, M H Fritz, N F Hansen, E Y Durand, A S Malaspinas, J D Jensen, T Marques-Bonet, C Alkan, K Prüfer, M Meyer, H A Burbano, J M Good, R Schultz, A Aximu-Petri, A Butthof, B Höber, B Höffner, M Siegemund, A Weihmann, C Nusbaum, E S Lander, C Russ, N Novod, J Affourtit, M Egholm, C Verna, P Rudan, D Brajkovic, Z Kucan, I Gusic, V B Doronichev, L V Golovanova, C Lalueza-Fox, M de la Rasilla, J Fortea, A Rosas, R W Schmitz, P L Johnson, E E Eichler, D Falush, E Birney, J C Mullikin, M Slatkin, R Nielsen, J Kelso, M Lachmann, D Reich, and S Pääbo. A draft sequence of the Neandertal genome. Science, 328 (5979):710-722, May 2010. doi: 10.1126/science.1188021. URL http://www.ncbi.nlm.nih.gov/pubmed/ 20448178.

Robert C. Griffiths and Paul Marjoram. An ancestral recombination graph. In Progress in population genetics and human evolution (Minneapolis, MN, 1994), volume 87 of IMA Vol. Math. Appl., pages 257-270. Springer, New York, 1997. URL http://www.math. canterbury.ac.nz/ r. sainudiin/recomb/ima.pdf.

Wolfgang Haak, Iosif Lazaridis, Nick Patterson, Nadin Rohland, Swapan Mallick, Bastien Llamas, Guido Brandt, Susanne Nordenfelt, Eadaoin Harney, Kristin Stewardson, Qiaomei Fu, Alissa Mittnik, Eszter Bánffy, Christos Economou, Michael Francken, Susanne Friederich, Rafael Garrido Pena, Fredrik Hallgren, Valery Khartanovich, Aleksandr Khokhlov, Michael Kunst, Pavel Kuznetsov, Harald Meller, Oleg Mochalov, Vayacheslav Moiseyev, Nicole Nicklisch, Sandra L. Pichler, Roberto Risch, Manuel A. Rojo Guerra, Christina Roth, Anna Szécsényi-Nagy, Joachim Wahl, Matthias Meyer, Johannes Krause, Dorcas Brown, David Anthony, Alan Cooper, Kurt Werner Alt, and David Reich. Massive migration from the steppe is a source for Indo-European languages in Europe. Nature, aop, March 2015. doi: 10.1101/013433. URL http://dx.doi.org/10.1038/nature14317.

Benjamin C. Haller, Jared Galloway, Jerome Kelleher, Philipp W. Messer, and Peter L. Ralph. Tree-sequence recording in SLiM opens new horizons for forward-time simulation of whole genomes. bioRxiv, 2018. doi: 10.1101/407783. URL https://www.biorxiv.org/content/early/2018/09/04/407783.

Richard R. Hudson. The variance of coalescent time estimates from DNA sequences. Journal of Molecular Evolution, 64(6):702-705, 2007. ISSN 0022-2844. doi: 10.1007/s00239-006-0261-1. URL http://dx. doi . org/10.1007/s00239-006-0261-1. 
Paul A. Jenkins and Yun S. Song. The effect of recurrent mutation on the frequency spectrum of a segregating site and the age of an allele. Theoretical Population Biology, 80(2):158 - 173, 2011. ISSN 0040-5809. doi: 10.1016/j.tpb.2011.04.001. URL http://www.sciencedirect.com/science/article/ $\mathrm{pii} / \mathrm{S} 0040580911000402$.

J Kelleher, A M Etheridge, and G McVean. Efficient coalescent simulation and genealogical analysis for large sample sizes. PLoS Comput Biol, 12(5), May 2016. doi: 10.1371/journal.pcbi.1004842. URL https: //www.ncbi.nlm.nih.gov/pubmed/27145223.

Jerome Kelleher, Kevin Thornton, Jaime Ashander, and Peter Ralph. Efficient pedigree recording for fast population genetics simulation. bioRxiv, 2018. doi: 10.1101/248500. URL https://www.biorxiv.org/ content/early/2018/06/07/248500.

J. F. C. Kingman. Poisson processes, volume 3 of Oxford Studies in Probability. The Clarendon Press Oxford University Press, New York, 1993. ISBN 0-19-853693-3. Oxford Science Publications.

Rob J. Kulathinal, Laurie S. Stevison, and Mohamed A. F. Noor. The genomics of speciation in Drosophila: Diversity, divergence, and introgression estimated using low-coverage genome sequencing. PLoS Genet, 5(7):e1000550, 07 2009. doi: 10.1371/journal.pgen.1000550. URL http://dx.doi.org/10. 1371\%2Fjournal.pgen. 1000550 .

Earl Lawrence, George Michailidis, Vijayan N Nair, and Bowei Xi. Network tomography: A review and recent developments. In Frontiers in Statistics, chapter 16, pages 345-366. World Scientific, July 2006. doi: 10. 1142/9781860948886_0016. URL http://www.stat.purdue.edu/ xbw/research/frontiers. 2006.pdf.

Mark Lipson, Po-Ru Loh, Sriram Sankararaman, Nick Patterson, Bonnie Berger, and David Reich. Calibrating the human mutation rate via ancestral recombination density in diploid genomes. bioRxiv, 2015 . doi: $10.1101 / 015560$.

Hugh McColl, Fernando Racimo, Lasse Vinner, Fabrice Demeter, Takashi Gakuhari, J. Víctor Moreno-Mayar, George van Driem, Uffe Gram Wilken, Andaine Seguin-Orlando, Constanza de la Fuente Castro, Sally Wasef, Rasmi Shoocongdej, Viengkeo Souksavatdy, Thongsa Sayavongkhamdy, Mohd Mokhtar Saidin, Morten E. Allentoft, Takehiro Sato, Anna-Sapfo Malaspinas, Farhang A. Aghakhanian, Thorfinn Korneliussen, Ana Prohaska, Ashot Margaryan, Peter de Barros Damgaard, Supannee Kaewsutthi, Patcharee Lertrit, Thi Mai Huong Nguyen, Hsiao-chun Hung, Thi Minh Tran, Huu Nghia Truong, Giang Hai Nguyen, Shaiful Shahidan, Ketut Wiradnyana, Hiromi Matsumae, Nobuo Shigehara, Minoru Yoneda, Hajime Ishida, Tadayuki Masuyama, Yasuhiro Yamada, Atsushi Tajima, Hiroki Shibata, Atsushi Toyoda, Tsunehiko Hanihara, Shigeki Nakagome, Thibaut Deviese, Anne-Marie Bacon, Philippe Duringer, Jean-Luc Ponche, Laura Shackelford, Elise Patole-Edoumba, Anh Tuan Nguyen, Bérénice Bellina-Pryce, Jean-Christophe Galipaud, Rebecca Kinaston, Hallie Buckley, Christophe Pottier, Simon Rasmussen, Tom Higham, Robert A. Foley, Marta Mirazón Lahr, Ludovic Orlando, Martin Sikora, Maude E. Phipps, Hiroki Oota, Charles Higham, David M. Lambert, and Eske Willerslev. The prehistoric peopling of Southeast Asia. Science, 361(6397):88-92, 2018. ISSN 0036-8075. doi: 10.1126/science.aat3628. URL http://science.sciencemag. org/content/361/6397/88.

G A McVean. A genealogical interpretation of linkage disequilibrium. Genetics, 162(2):987-991, October 2002. URL http://www.ncbi.nlm.nih.gov/pmc/articles/PMC1462283/.

Gil McVean. A genealogical interpretation of principal components analysis. PLoS Genet, 5(10):e1000686, 10 2009. doi: 10.1371/journal.pgen.1000686. URL http://dx.doi.org/10.1371\%2Fjournal.pgen. 1000686.

K Misawa and R F Kikuno. Evaluation of the effect of CpG hypermutability on human codon substitution. Gene, 431(1-2):18-22, February 2009. doi: 10.1016/j.gene.2008.11.006. URL http://www.ncbi.nlm.nih. gov/pubmed/19059467. 
Jian Ni and S. Tatikonda. Network tomography based on additive metrics. Information Theory, IEEE Transactions on, 57(12):7798-7809, December 2011. ISSN 0018-9448. doi: 10.1109/TIT.2011.2168901. URL http://arxiv.org/pdf/0809.0158.

N Patterson, P Moorjani, Y Luo, S Mallick, N Rohland, Y Zhan, T Genschoreck, T Webster, and D Reich. Ancient admixture in human history. Genetics, 192(3):1065-1093, November 2012. doi: 10.1534/genetics. 112.145037. URL http://www.ncbi.nlm.nih.gov/pubmed/22960212.

Giovanni Peccati and Murad S. Taqqu. Some facts about Charlier polynomials. In Wiener Chaos: Moments, Cumulants and Diagrams, volume 1 of Bocconi \& Springer Series, pages 171-175. Springer Milan, 2011. ISBN 978-88-470-1678-1. doi: 10.1007/978-88-470-1679-8_10. URL http://dx.doi.org/10.1007/ 978-88-470-1679-8_10.

Benjamin M. Peter. Admixture, population structure, and F-statistics. Genetics, 202(4):1485-1501, 2016. URL http://www.genetics.org/content/202/4/1485. abstract.

Peter Ralph and Graham Coop. The geography of recent genetic ancestry across Europe. PLoS Biol, 11(5): e1001555, 05 2013. doi: 10.1371/journal.pbio.1001555. URL http://dx.doi.org/10.1371\%2Fjournal. pbio. 1001555.

David Reich, Richard E. Green, Martin Kircher, Johannes Krause, Nick Patterson, Eric Y. Durand, Bence Viola, Adrian W. Briggs, Udo Stenzel, Philip L. F. Johnson, Tomislav Maricic, Jeffrey M. Good, Tomas Marques-Bonet, Can Alkan, Qiaomei Fu, Swapan Mallick, Heng Li, Matthias Meyer, Evan E. Eichler, Mark Stoneking, Michael Richards, Sahra Talamo, Michael V. Shunkov, Anatoli P. Derevianko, Jean-Jacques Hublin, Janet Kelso, Montgomery Slatkin, and Svante Paabo. Genetic history of an archaic hominin group from Denisova cave in Siberia. Nature, 468(7327):1053-1060, December 2010. ISSN 00280836. URL http://dx.doi.org/10.1038/nature09710.

Charles Semple and Mike Steel. Phylogenetics. 2003.

Harsh Singhal and George Michailidis. Identifiability of flow distributions from link measurements with applications to computer networks. Inverse Problems, 23(5):1821, 2007. URL http://stacks.iop.org/ $0266-5611 / 23 / i=5 / a=004$.

M Slatkin. Inbreeding coefficients and coalescence times. Genet Res, 58(2):167-175, October 1991. URL http://www.ncbi.nlm.nih.gov/pubmed/1765264.

Doug Speed and David Balding. Relatedness in the post-genome era: is it still useful? Nature Reviews Genetics, 16:33-44, 2014. doi: doi:10.1038/nrg3821. URL http://www.nature.com/nrg/journal/v16/ $\mathrm{n} 1 / \mathrm{full} / \mathrm{nrg} 3821$.html.

F Tajima. Evolutionary relationship of dna sequences in finite populations. Genetics, 105(2):437-460, October 1983. URL http://www.ncbi.nlm.nih.gov/pmc/articles/PMC1202167/.

H Tang, D O Siegmund, P Shen, P J Oefner, and M W Feldman. Frequentist estimation of coalescence times from nucleotide sequence data using a tree-based partition. Genetics, 161(1):447-459, May 2002. URL http://www.ncbi.nlm.nih.gov/pmc/articles/PMC1462078/.

A R Templeton, E Routman, and C A Phillips. Separating population structure from population history: a cladistic analysis of the geographical distribution of mitochondrial DNA haplotypes in the tiger salamander, ambystoma tigrinum. Genetics, 140(2):767-782, June 1995. URL https://www.ncbi.nlm.nih.gov/ pubmed/7498753.

R Thomson, J K Pritchard, P Shen, P J Oefner, and M W Feldman. Recent common ancestry of human Y chromosomes: evidence from DNA sequence data. Proc Natl Acad Sci U S A, 97(13):7360-7365, June 2000. URL http://www.ncbi.nlm.nih.gov/pubmed/10861004. 
L Vigilant, M Stoneking, H Harpending, K Hawkes, and A C Wilson. African populations and the evolution of human mitochondrial DNA. Science, 253(5027):1503-1507, September 1991. URL https://www.ncbi . nlm.nih.gov/pubmed/1840702.

J Wakeley, L King, B S Low, and S Ramachandran. Gene genealogies within a fixed pedigree, and the robustness of Kingman's coalescent. Genetics, 190(4):1433-1445, April 2012. doi: 10.1534/genetics.111. 135574. URL http://www.ncbi.nlm.nih.gov/pubmed/22234858.

G.A. Watterson. On the number of segregating sites in genetical models without recombination. Theoretical Population Biology, 7(2):256 - 276, 1975. ISSN 0040-5809. doi: http://dx.doi.org/10.1016/0040-5809(75) 90020-9. URL http://www. sciencedirect.com/science/article/pii/0040580975900209.

Sewall Wright. Coefficients of inbreeding and relationship. The American Naturalist, 56(645):pp. 330-338, 1922. ISSN 00030147. URL http://www.jstor.org/stable/2456273.

\section{The variance of $C_{x, y}$}

Continuation of proof of Theorem 2. First note that (43) implies that

$$
C_{x, y}=\frac{1}{n} \sum_{j=1}^{n} \sum_{k=1}^{n} Z_{j k}\left(\delta_{j k}-\frac{1}{n}\right),
$$

where $\delta_{j k}=1$ if $j=k$ and is zero otherwise. To find the variance of $C_{x, y}$, we need to compute covariances of the $Z_{j k}$ terms. To do this, it is most efficient to record the general case. The following lemma follows directly from Lemma 3, which uses upper-case roman characters for test functions to make the result more visually intuitive and easier to apply.

Lemma 4. If $A, B, C$, and $D$ are test functions as in Lemma 3, then

$$
\operatorname{cov}[M(A) M(B), M(C)]=\mu(A B C)+\mu(A) \mu(B C)+\mu(B) \mu(A C)
$$

and

$$
\begin{aligned}
\operatorname{cov}[M(A) M(B), M(C) M(D)]=\mu & A B C D)+\mu(A) \mu(B C D)+\mu(B) \mu(A C D)+\mu(C) \mu(A B D) \\
& +\mu(D) \mu(A B C)+\mu(A C) \mu(B D)+\mu(A D) \mu(B C) \\
& +\mu(A) \mu(C) \mu(B D)+\mu(A) \mu(D) \mu(B C) \\
& +\mu(A C) \mu(B) \mu(D)+\mu(A D) \mu(B) \mu(C)
\end{aligned} .
$$

If $j \neq k$, then $\psi_{j, x}$ and $\psi_{k, x}$ are supported on disjoint parts of the ancestral genomes, and so $\psi_{j, x} \psi_{k, y}=0$. For the same reason, the covariance of $Z_{j k}$ and $Z_{\ell m}$ is zero unless at least one of $j$ and $k$ match at least one of $\ell$ and $m$. We are further helped by the fact that $\psi_{j, x}^{n}=\psi_{j, x} / W_{j}^{n-1}$.

First, we apply Lemma 4 with $A=C=\psi_{j, x}$ and $B=D=\psi_{k, y}$ and $j \neq k$ (so, note that $A B=A D=$ $C B=C D=0$ ).

$$
\operatorname{var}\left[Z_{j k}\right]=\frac{\mu\left(\psi_{j, x}\right) \mu\left(\psi_{k, y}\right)}{\left|W_{j}\right|\left|W_{k}\right|}\left\{1+\left|W_{j}\right| \mu\left(\psi_{j, x}\right)+\left|W_{k}\right| \mu\left(\psi_{k, y}\right)\right\}
$$

Similarly, if $A=\psi_{j, x}, B=\psi_{k, y}, C=\psi_{k, x}$, and $D=\psi_{j, y}$, and $j \neq k$ (so, note that $A B=A C=B D=$ $C D=0$ ).

$$
\operatorname{cov}\left[Z_{j k}, Z_{k j}\right]=\mu\left(\psi_{j, x} \psi_{j, y}\right) \mu\left(\psi_{k, y} \psi_{k, x}\right)+\mu\left(\psi_{j, x}\right) \mu\left(\psi_{j, y}\right) \mu\left(\psi_{k, y} \psi_{k, x}\right)+\mu\left(\psi_{j, x} \psi_{j, y}\right) \mu\left(\psi_{k, y}\right) \mu\left(\psi_{k, x}\right)
$$


Now, with $A=C=\psi_{j, x}, B=\psi_{k, y}$, and $C=\psi_{l, y}$, and $j, k$ and $l$ all distinct,

$$
\operatorname{cov}\left[Z_{j k}, Z_{j l}\right]=\frac{1}{W_{j}} \mu\left(\psi_{j, x}\right) \mu\left(\psi_{k, y}\right) \mu\left(\psi_{l, y}\right)
$$

and similarly,

$$
\operatorname{cov}\left[Z_{k j}, Z_{j l}\right]=\mu\left(\psi_{j, x} \psi_{j, y}\right) \mu\left(\psi_{k, x}\right) \mu\left(\psi_{l, y}\right) .
$$

Now, with $A=C=\psi_{j, x}, B=\psi_{j, y}$, and $D=\psi_{k, y}$,

$$
\operatorname{cov}\left[M\left(\psi_{j, x}\right) M\left(\psi_{j, y}\right), M\left(\psi_{j, x}\right) M\left(\psi_{k, y}\right)\right]=\mu\left(\psi_{k, y}\right)\left\{\mu\left(\psi_{j, x} \psi_{j, y}\right)\left(\frac{1}{W_{j}}+\mu\left(\psi_{j, x}\right)\right)+\frac{1}{W_{j}} \mu\left(\psi_{j, x}\right) \mu\left(\psi_{j, y}\right)\right\}
$$

and with $A=\psi_{j, x}, B=\psi_{k, y}$, and $C=\psi_{j, x} \psi_{j, y}$,

$$
\operatorname{cov}\left[M\left(\psi_{j, x}\right) M\left(\psi_{k, y}\right), M\left(\psi_{j, x} \psi_{j, y}\right)\right]=\frac{1}{W_{j}} \mu\left(\psi_{k, y}\right) \mu\left(\psi_{j, x} \psi_{j, y}\right) .
$$

These combine to give

$$
\operatorname{cov}\left[Z_{j j}, Z_{j k}\right]=\mu\left(\psi_{k, y}\right)\left\{\mu\left(\psi_{j, x} \psi_{j, y}\right) \mu\left(\psi_{j, x}\right)+\frac{1}{W_{j}} \mu\left(\psi_{j, x}\right) \mu\left(\psi_{j, y}\right)\right\}
$$

Finally, taking $A=C=\psi_{j, x}$ and $B=D=\psi_{j, y}$, we get

$$
\begin{aligned}
\operatorname{var}\left[M\left(\psi_{j, x}\right) M\left(\psi_{j, y}\right)\right]= & \frac{1}{W_{j}^{2}} \mu\left(\psi_{j, x} \psi_{j, y}\right)+\frac{2}{W_{j}} \mu\left(\psi_{j, x} \psi_{j, y}\right)\left(\mu\left(\psi_{j, x}\right)+\mu\left(\psi_{j, y}\right)\right) \\
& +\frac{1}{W_{j}^{2}} \mu\left(\psi_{j, x}\right) \mu\left(\psi_{j, y}\right)+\mu\left(\psi_{j, x} \psi_{j, y}\right)^{2}+\frac{1}{W_{j}} \mu\left(\psi_{j, x}\right)^{2} \mu\left(\psi_{j, y}\right) \\
& +\frac{1}{W_{j}} \mu\left(\psi_{j, x}\right) \mu\left(\psi_{j, y}\right)^{2}+2 \mu\left(\psi_{j, x}\right) \mu\left(\psi_{j, y}\right) \mu\left(\psi_{j, y} \psi_{j, x}\right)
\end{aligned}
$$

and

$$
\operatorname{cov}\left[M\left(\psi_{j, x}\right) M\left(\psi_{j, y}\right), M\left(\psi_{j, x} \psi_{j, y}\right)\right]=\frac{1}{W_{j}^{2}} \mu\left(\psi_{j, x} \psi_{j, y}\right)+\frac{1}{W_{j}} \mu\left(\psi_{j, x} \psi_{j, y}\right)\left(\mu\left(\psi_{j, x}\right)+\mu\left(\psi_{j, y}\right)\right.
$$

and

$$
\operatorname{var}\left[M\left(\psi_{j, x} \psi_{j, y}\right)\right]=\frac{1}{W_{j}^{2}} \mu\left(\psi_{j, x} \psi_{j, y}\right)
$$

so

$$
\begin{aligned}
\operatorname{var}\left[Z_{j j}\right] & =\operatorname{var}\left[M\left(\psi_{j, x}\right) M\left(\psi_{j, y}\right)\right]-2 \operatorname{cov}\left[M\left(\psi_{j, x}\right) M\left(\psi_{j, y}\right), M\left(\psi_{j, x} \psi_{j, y}\right)\right]+\operatorname{var}\left[M\left(\psi_{j, x} \psi_{j, y}\right)\right] \\
& =\mu\left(\psi_{j, x}\right) \mu\left(\psi_{j, y}\right)\left(\frac{1}{W_{j}^{2}}+\frac{1}{W_{j}}\left(\mu\left(\psi_{j, x}\right)+\mu\left(\psi_{j, y}\right)\right)+2 \mu\left(\psi_{j, y} \psi_{j, x}\right)\right)+\mu\left(\psi_{j, y} \psi_{j, x}\right)^{2}
\end{aligned}
$$


We can put these together to obtain that

$$
\begin{aligned}
& \operatorname{var}\left[C_{x, y}\right]=\frac{1}{n^{2}} \sum_{1 \leq j, k, \ell, m \leq n} \operatorname{cov}\left[Z_{j k}, Z_{\ell m}\right]\left(\delta_{j k}-\frac{1}{n}\right)\left(\delta_{\ell m}-\frac{1}{n}\right) \\
& =\frac{(n-1)^{2}}{n^{4}} \sum_{j} \operatorname{var}\left[Z_{j j}\right]-\frac{2(n-1)}{n^{4}} \sum_{j \neq k} \operatorname{cov}\left[Z_{j j}, Z_{j k}+Z_{k j}\right]+\frac{1}{n^{4}} \sum_{j \neq k} \operatorname{var}\left[Z_{j k}\right] \\
& +\frac{1}{n^{4}} \sum_{j \neq k} \operatorname{cov}\left[Z_{j k}, Z_{k j}\right]+\frac{1}{n^{4}} \sum_{j \neq k \neq \ell \neq j} \operatorname{cov}\left[Z_{j k}, Z_{j \ell}\right] \\
& +\frac{1}{n^{4}} \sum_{j \neq k \neq \ell \neq j} \operatorname{cov}\left[Z_{k j}, Z_{\ell j}\right]+\frac{2}{n^{4}} \sum_{j \neq k \neq \ell \neq j} \operatorname{cov}\left[Z_{k j}, Z_{j \ell}\right] \\
& =\frac{(n-1)^{2}}{n^{4}} \sum_{j}\left(\mu\left(\psi_{j, x}\right) \mu\left(\psi_{j, y}\right)\left(\frac{1}{W_{j}^{2}}+\frac{1}{W_{j}}\left(\mu\left(\psi_{j, x}\right)+\mu\left(\psi_{j, y}\right)\right)+2 \mu\left(\psi_{j, y} \psi_{j, x}\right)\right)+\mu\left(\psi_{j, y} \psi_{j, x}\right)^{2}\right) \\
& -\frac{2(n-1)}{n^{4}} \sum_{j \neq k}\left(\mu\left(\psi_{k, y}\right)\left\{\mu\left(\psi_{j, x} \psi_{j, y}\right) \mu\left(\psi_{j, x}\right)+\frac{1}{W_{j}} \mu\left(\psi_{j, x}\right) \mu\left(\psi_{j, y}\right)\right\}\right. \\
& \left.+\mu\left(\psi_{k, x}\right)\left\{\mu\left(\psi_{j, y} \psi_{j, x}\right) \mu\left(\psi_{j, y}\right)+\frac{1}{W_{j}} \mu\left(\psi_{j, y}\right) \mu\left(\psi_{j, x}\right)\right\}\right) \\
& +\frac{2}{n^{4}} \sum_{j \neq k}\left(\frac{1}{W_{j}} \mu\left(\psi_{j, x}\right) \mu\left(\psi_{k, y}\right)\left\{\frac{1}{W_{j}}+\mu\left(\psi_{j, x}\right)+\mu\left(\psi_{k, y}\right)\right\}\right) \\
& +\frac{1}{n^{4}} \sum_{j \neq k}\left(\mu\left(\psi_{j, x} \psi_{j, y}\right) \mu\left(\psi_{k, y} \psi_{k, x}\right)+\mu\left(\psi_{j, x}\right) \mu\left(\psi_{j, y}\right) \mu\left(\psi_{k, y} \psi_{k, x}\right)+\mu\left(\psi_{j, x} \psi_{j, y}\right) \mu\left(\psi_{k, y}\right) \mu\left(\psi_{k, x}\right)\right) \\
& +\frac{1}{n^{4}} \sum_{j \neq k \neq \ell \neq j} \frac{1}{W_{j}} \mu\left(\psi_{j, x}\right) \mu\left(\psi_{k, y}\right) \mu\left(\psi_{l, y}\right) \\
& +\frac{1}{n^{4}} \sum_{j \neq k \neq \ell \neq j} \frac{1}{W_{j}} \mu\left(\psi_{j, y}\right) \mu\left(\psi_{k, x}\right) \mu\left(\psi_{l, x}\right) \\
& +\frac{2}{n^{4}} \sum_{j \neq k \neq \ell \neq j} \mu\left(\psi_{j, x} \psi_{j, y}\right) \mu\left(\psi_{k, x}\right) \mu\left(\psi_{l, y}\right)
\end{aligned}
$$

The above is dominated by terms of the form $(\overline{\mu t})^{3} / n|W|$, where $\overline{\mu t}$ is an average of $\mu(\psi$.) terms. To put a crude bound on this, assume that $\mu\left(\psi_{j, x}\right)$ and $\mu\left(\psi_{j, y}\right)$ are bounded by $\epsilon$ for every $j$, resulting in $\operatorname{var}\left[C_{x, y}\right] \leq \frac{\epsilon^{3}}{n|W|}$, as in the theorem. 